\title{
HISTORIA RECIENTE DE LA UNIVERSIDAD DE ALCALÁ (CONTINUACIÓN)
}

\section{RECENT HISTORY OF THE UNIVERSITY OF ALCALA (CONTINUED)}

\author{
AUTORES \\ José Morilla Critz: Catedrático de Universidad. Área de Fundamentos de la \\ Economía. \\ jose.morilla@ozu.es
}

\section{CURRÍCULUM VITAE}

Profesor en la Facultad de Ciencias Económicas y Empresariales. Universidad de Alcalá

\section{RESUMEN}

La consecuencia de la desaparición de la tímida política de planificación universitaria que se había llevado a cabo en la etapa de Villar Palasí, en unos años que iban a registrar la verdadera avalancha de solicitudes para entrar en la Universidad, dio como resultado que, para Madrid, los temas de ampliación de la dotación de medios físicos y humanos para atender la enseñanza universitaria fueran objeto de continuas, y urgentes cada año, negociaciones entre las autoridades universitarias, principalmente de la Complutense, y el Ministerio de Educación. De este modo, la función de "planificación", que parece le debía caber al Ministerio, se suplió por las ideas que el equipo de gobierno de la Complutense desarrollase para alojar cada año un número creciente de estudiantes. 
Alcalá - Universidad - Historia - Proyectos

\begin{abstract}
The consequence of the disappearance of the timid university planning policy was carried out in Villar Palasí stage in a few years they would record the avalanche of applications to enter the university, which resulted to Madrid, issues of expansion of the provision of physical and human resources to attend university education were subject to continuous and urgent every year, negotiations between the university authorities, mainly from the Complutense University and the Ministry of Education. Thus, the role of "planning," it seems you had to fit to the Ministry, was supplied by the ideas that the team develop Complutense government each year to accommodate increasing numbers of students.
\end{abstract}

\title{
KEY WORDS
}

Alcala - University - History - Projects

Para este capítulo, José Morilla nos trae a la memoria el lento y penoso proceso de auténtica resurrección de la Universidad de Alcalá, como si tratara de un bebé nacido con graves deficiencias psíquicas y físicas, comenzado en 1975 y culminado con la publicación del Real Decreto de 10 de junio de 1977, por el que se crea "una nueva Universidad de Madrid con sede en Alcalá de Henares".

\section{EL RENACIMIENTO DE LA UNIVERSIDAD DE ALCALÁ}




\section{1975-1977: un proyecto de reapertura al fin con éxito}

La consecuencia de la desaparición de la tímida política de planificación universitaria que se había llevado a cabo en la etapa de Villar Palasí, en unos años que iban a registrar la verdadera avalancha de solicitudes para entrar en la Universidad, dio como resultado que, para Madrid, los temas de ampliación de la dotación de medios físicos y humanos para atender la enseñanza universitaria fueran objeto de continuas, y urgentes cada año, negociaciones entre las autoridades universitarias, principalmente de la Complutense, y el Ministerio de Educación. De este modo, la función de "planificación", que parece le debía caber al Ministerio, se suplió por las ideas que el equipo de gobierno de la Complutense desarrollase para alojar cada año un número creciente de estudiantes.

En el curso 1974-1975 las Universidades de Madrid tenían 129.549 alumnos matriculados, de los cuales 89.667 lo estaban en la "Complutense" y a mediados de curso se calculaba ya que para el próximo se rebasarían los 100.000 alumnos. La política de colegios mayores improvisados no solventaba en nada la masificación, porque, por una parte, seguía llenando las facultades en los últimos cursos y, por otra, docente y administrativamente no descongestionaba el funcionamiento de departamentos y órganos gestores. Además, tal política se había adoptado como situación provisional, lo que desde el punto de vista de la inversión y otros gastos era un verdadero derroche para una situación precaria. En tal situación, no tiene nada de extraño que el Rectorado de la "Complutense", una vez archivado el peregrino proyecto de Julio Rodríguez, desempolvara la idea de 1972: desdoblar alguna de sus facultades más masificadas aprovechando las instalaciones de Alcalá de Henares.

Al tiempo que la Dirección General de Enseñanzas Medias pretendía rentabilizar la pequeña inversión realizada en el acondicionamiento de edificaciones de la Base 
Aérea de Alcalá, con la instalación "provisional" del Instituto, la Universidad Complutense, desde mayo de 1975, negociaba con la Dirección General de Universidades y con el Ayuntamiento de Alcalá la posibilidad de abrir en dichas instalaciones los primeros cursos de unas nuevas Facultades de Químicas, Biología y Ciencias Económicas y Empresariales.

Esta vez el acuerdo entre la Universidad madrileña y el Ministerio parecía definido, de tal modo que para el "Campus" de Alcalá el segundo hizo público, en agosto de dicho añoNota24, que la creación de las mencionadas facultades se efectuaría mediante un decreto del Ministerio que, a su vez, dotaría las plazas de catedráticos, profesores agregados y adjuntos necesarios para las clases del primer curso de las correspondientes carreras. Por otra parte, unos días antes, la Junta de Construcciones del MEC hizo copartícipe a la Universidad Complutense en el uso de los terrenos de la Base Aérea y encargó a un equipo de arquitectos la realización de un Plan de ordenación de los mismos como campus universitarioNota25.

Es interesante destacar que, con ocasión de las visitas que las delegaciones del equipo de gobierno de la "Complutense" hicieron a Alcalá para reunirse con las autoridades locales, la ciudad tuvo la oportunidad de mostrar la opción "recuperadora" universitaria en el casco antiguo, junto a la alternativa del "Campus".

La ciudad mostró la opción acompañándola de ofrecimiento de edificios históricos: dos por parte del Ayuntamiento (convento de la Madre de Dios y Colegio de Mínimos o antiguo Hospital Militar) y otros dos por la Sociedad de Condueños (Colegio de San Pedro y San Pablo y Colegio de la Madre de Dios) y proponiendo la constitución de instituciones comunes (en concreto el Museo Cisneros) Nota5 de VA.

El resultado de ese conocimiento fue la elaboración de tres informes por parte del vicerrector de los campi de la Complutense, Felipe Calvo, para su Junta de Gobierno, 
que recogen, a nuestro entender por primera vez, un conjunto de proposiciones articuladas en torno a la idea central de recuperar íntegramente Alcalá como ciudad universitaria, hasta tal punto que el propio Calvo llegó a llamar a las mismas en uno de esos informes con el significativo nombre de "operación Alcalá"Nota26.

Esta "operación Alcalá", concebida, no cabe duda, personalmente por el profesor Calvo, se proponía, resumidamente, los siguientes objetivos:

1. Crear en Alcalá un "Campus" de la Universidad Complutense de Madrid, compuesto por Facultades independientes de las homónimas existentes en Madrid, que llegara a alcanzar, cuando estuviera en pleno funcionamiento, los 20.000 alumnos.

2. En el plazo inmediato, el "Campus de Alcalá" se constituiría absorbiendo la mayor parte de las enseñanzas que se venían impartiendo en el "Colegio Universitario de Arcos de Jalón" y enviando también estudiantes de otras carreras como Medicina y Farmacia, comenzando el curso 1975-1976 con unos 5.000 estudiantes.

3. La especialización de ese campus sería la de formar fundamentalmente docentes en las diferentes materias, tanto para la enseñanza media como la superior, y la estructura de funcionamiento sería departamentalNota6 de VA.

4. La base física del campus sería, por una parte, un campus nuevo completo a construir (facultades, instalaciones complementarias, residencias y jardín botánico) en la antigua base aérea y un conjunto universitario urbano constituido por los antiguos edificios universitarios de Alcalá, recuperados dentro de un "Plan de ordenación urbana universitaria" convenido con diversas administraciones públicas y privadasNota7 de VA. 
5. Recuperación del Colegio Mayor de San Ildefonso como símbolo más representativo, a cambio de ceder instalaciones de la Universidad Complutense en Madrid para sede de la Escuela de Administración Pública.

Este plan era, ciertamente, muy coherente y ambicioso y hubiera necesitado para llevarse a cabo la asunción clara y definida tanto de la Junta de Gobierno de la Complutense como del Ministerio de Educación, de los que solamente nos consta su atención pasiva.

Para los responsables del Ministerio de Educación, y parece que también para el órgano de gobierno colegiado de la Universidad de Madrid, lo que realmente había sobre la mesa en el verano de 1975 era:

por una parte, una inmensa extensión de terreno prácticamente sin urbanizar pero propiedad del Estado, unas instalaciones de 5.000 metros cuadrados (antiguo cuartelNota8de VA) semiacondicionadas, una promesa de rehabilitación de un edificio histórico en la ciudad de 3.000 metros cuadrados y por otra, unos 5.000 estudiantes "sobrantes" que había que ubicar urgentemente en cualquier sitio.

Pero ambos hechos juntos creemos que determinaron, al fin, que en Alcalá de Henares se reabriera la enseñanza universitaria, en octubre de 1975.

La apariencia de existencia de un plan facilitó adoptar la decisión de enviar al "Campus de Alcalá" los alumnos que ese año se habían matriculado en el Colegio Universitario de "Arcos de Jalón" (Casa de Campo para Económicas y Empresariales, y barrio de San Blas para Químicas y Biológicas) y los sobrantes de Medicina y Farmacia que, en total, eran unos 1.500. A corto plazo, sin embargo, prácticamente no se realizó ninguna inversión más en instalaciones y equipamiento. Los equipos de mantenimiento de la Complutense limpiaron algo de vegetación los accesos a la base 
aérea y rehabilitaron provisionalmente algunos barracones abandonados en la misma, se rescataron algunas balanzas de la chatarra de la Facultad de Químicas de la Moncloa, se trasladaron algunos libros de la Complutense, se negoció con RENFE la construcción de un apeadero, y poco másNota9 de VA.

Pero el principal vicio de esta "reapertura" fue que la enseñanza (con algunos profesores trasladados de la Complutense, venidos otros de otras universidades en comisión de servicios, contratados por aquélla) quedó indefinida en cuanto a su dependencia orgánica y su status jurídico. Las facultades previstas, al comenzar las clases, no se habían creado oficialmente y, sin embargo, no eran una extensión de las respectivas facultades y departamentos de la Universidad Complutense. De este modo se puede decir que la Universidad de Alcalá se abrió en octubre de 1975 sin instalaciones, sin existir jurídicamente, sin ser una extensión orgánica de ninguna otra, pero con 1.500 estudiantes que se habían inscrito previamente sin saber que desde Madrid serían enviados por sorpresa a Alcalá.

Si quisiéramos encontrar referente histórico a esta forma tan humilde e irregular de reabrir una universidad en el mismo lugar en que había existido una de las más grandes de España en otro tiempo, nos acordaríamos de la frase de Cisneros cuando dijo aquello de que "otros harán en piedra lo que yo hice en barro".

Pero hay que tener en cuenta que, en aquel momento, ni tan siquiera de esta forma había quedado asegurado que la enseñanza universitaria en Alcalá se iba a reabrir definitivamente. No habiéndose adoptado ninguna decisión legal concreta al respecto, el extraordinario cambio que iba a significar para la Administración la muerte de Franco en noviembre de 1975 -precisamente a las pocas semanas de haberse comenzado las clases en el "Campus de Alcalá"-, dejó completamente en el aire los acuerdos verbales que habían existido entre el Ministerio de Educación y la 
Universidad Complutense. Sin embargo, se había creado la situación de hecho de 1.500 estudiantes en aquel campus Nota10 de VA.

La muerte del dictador, el primer discurso del rey en las Cortes, las intenciones declaradas de los más importantes miembros del Gobierno Arias y, en fin, la propia dinámica de las circunstancias, crearon en el país la lógica esperanza por un cambio que atendiera a las reivindicaciones que había pendientes en muchos sectores sociales. Dentro de ellas estaban las de los universitarios, tanto en su versión de reivindicaciones estudiantiles (eminentemente políticas), como de los profesores más jóvenes (profesionales y políticos). Los universitarios habían sido uno de los principales generadores de movimientos de oposición al régimen franquista y de formación de cuadros democráticos, y tras la muerte de su titular se colocaron a la cabeza de la exigencia de "la ruptura democrática" y de la inmediata satisfacción de las reivindicaciones pendientes.

En tales circunstancias, la débil situación jurídica, la carencia casi absoluta de medios para el desenvolvimiento de la enseñanza en la que se encontró el "Campus" de Alcalá y la sospecha de adscripción de sus principales responsables a grupos de presión conservadores (eminentemente el Opus Dei), no tenía por menos que poner en peligro la continuación del proyecto de Alcalá, como así fue.

A las pocas semanas de comenzarse las clases en el "Campus", los estudiantes, sobre todo los de las facultades más numerosas (Económicas y Empresariales y Medicina), empezaron a manifestar su oposición a venir a Alcalá (la gran mayoría eran de Madrid). El malestar era absolutamente justo, no había instalaciones adecuadas, no existía biblioteca, ni comedor, ni salas de lectura, ni instalaciones deportivas, ni despachos de profesores... 
En el corto plazo, el desarrollo del "Campus" de Alcalá iba a estar condicionado, pues, por un conjunto de circunstancias que podemos clasificar entre las siguientes categorías:

1. La despreocupación del Ministerio por el proyecto.

2. La desautorización política de los responsables iniciales del proyecto entre la comunidad estudiantil y parte del profesorado, constituida ya en el "Campus" de Alcalá.

3. La desautorización, de hecho, del "Campus" por los departamentos de la Universidad Complutense.

Era, hasta cierto punto, lógico que el Ministerio de Educación se desentendiera del proyecto de enseñanza universitaria en Alcalá dada la situación política del momento. Es un hecho conocido que la universidad, salvo en cuanto a evitar en la medida de lo posible que en ella estallaran conflictos incontrolados (cosa que no se consiguió del todo en los primeros momentos), no formaba parte de las prioridades de actuación del primer gobierno. Incluso, en los gobiernos de la transición de UCD, no fue un tema de la urgencia de otros como la reforma política, el desmantelamiento de los sindicatos verticales, la organización autonómica del Estado, la disolución de los medios de comunicación del movimiento, etc. Por otra parte, una vez asumida la realidad de la crisis económica, era lógico que los recortes de la inversión pública, dadas las circunstancias señaladas, se manifestaran en la paralización de compromisos no formalizados, como era el del "Campus" de Alcalá.

En febrero de 1976 se había ultimado la redacción del "Plan parcial de ordenación del Campus de Alcalá", que fue presentado durante una Junta de Gobierno de la Universidad de Madrid, reunida extraordinariamente en Alcalá. En ella se hicieron 
previsiones, ante las autoridades locales invitadas, de un campus que alcanzaría los 25.000 estudiantes; pero ya se supo en esa misma reunión el silencio del nuevo Ministerio en cuanto a la solicitud hecha en su día de creación oficial de las correspondientes facultades. Una contestación afirmativa hubiera significado su dotación de presupuesto.

Era también lógico el desentendimiento de los departamentos de la Universidad de Madrid por el proyecto de Alcalá. De entrada, hay que decir que la universidad española ha sido y es una corporación en la que las autoridades centrales (fundamentalmente el Rectorado) tiene menos capacidad de decisión de hecho de la que jurídicamente puede parecer. Por entonces, lo que se llamaban "las cátedras" (que usufructuaban de hecho el poder institucional que correspondía a unos "departamentos" que, estando constituidos dentro de las facultades, se confundían con las "cátedras") y, dentro de ellas, sus titulares, eran las que condicionaban completamente la carrera profesional de los profesores y tenían en sus manos la calificación de los alumnos. Por esta vía, dado que la Universidad era y sigue siendo una institución gobernada por el profesorado (entonces por una minoría más o menos fiel al régimen, hoy por la entera corporaciónNota11 de VA), un proyecto tenía la virtualidad de ir adelante si contaba con el apoyo, o al menos no contaba con la resistencia de esas unidades "departamentales".

El proyecto de "Campus" en Alcalá había surgido directamente del Rectorado y el encargado de llevarlo a cabo, el profesor Felipe Calvo, pretendió poner en práctica aquellas ideas que ya vimos expuso en su informe a la Junta de Gobierno (estructura interdepartamental, facultades no orgánicas, especialización en la formación de docentes, etc.), con la seguridad de una inmediata creación oficial de los estudios por parte del Ministerio de Educación. De esta forma habría habido una total independencia de estudiantes y profesores con respecto a las cátedras, departamentos y facultades de Madrid. 
El deseo de independencia y de "proyecto autónomo y regeneracionista" con el que nacía este campus de la mano del citado vicerrector (de la Universidad de Madrid), quedó plasmado en la búsqueda de "decanos" para cada una de las facultades a crear que no pertenecieran a la Universidad Complutense. Fueron reclutados en comisión de servicios de otras universidades, con la particularidad de que, en una buena proporción, eran miembros o simpatizantes del Opus Dei. Lógicamente, el profesorado para comenzar los primeros cursos fue reclutado, por tales decanos, de igual forma, aun cuando aquí, dado el número, se produjo una gran variedad ideológica.

El caso fue que, en la mayor parte de los casos, las cátedras y departamentos de la Universidad Complutense fueron marginados del proceso de constitución del "Campus" de Alcalá. Ello tendría graves consecuencias en lo inmediato, una vez que no se crearon oficialmente las facultades, tal como se había previsto y, por tanto, la única legitimidad jurídica de las enseñanzas de Alcalá tenía que provenir de los departamentos y facultades correspondientes de la Universidad Complutense. Las enseñanzas de Alcalá, o eran ramas que les pertenecían, o no era nada. Sin embargo, surgido como había surgido el campus alcalaíno, rápidamente consideraron aquéllos que los alumnos y los profesores adscritos a Alcalá les correspondieran.

El "Campus" de Alcalá se encontró, pues, a poco de empezar (escasamente cuatro meses después), en una situación en la que el reducido grupo de "pioneros" (autoridades académicas del "Campus") se quedó sin soporte legal, económico ni institucional para continuar con el proyecto. Pero en él estaban involucrados un conjunto de profesores con contratos de un año en la mayoría de los casos, que habían venido de otras partes de España con la confianza de continuidad y, sobre todo, 1.500 estudiantes que, en su mayoría, habían sido trasladados contra su voluntad. 
En esa peligrosa situación hubiera bastado una actitud intransigente de los estudiantes con la situación que tenían que soportar, para que la enseñanza universitaria en Alcalá hubiera vuelto a desaparecer. Ciertamente hubo una gran contestación, pero creemos que el contenido político que entonces tenía el movimiento estudiantil y el encuentro que, en ese campo, había con los profesores "progresistas"Nota27, favoreció que la lucha estudiantil en Alcalá no se centrara exclusivamente en la exigencia de ser "devueltos" a Madrid (lo que, hablando en estrictos términos académicos, era la única posibilidad de garantizarles entonces unas mínimas condiciones legales y docentes).

Las protestas de los estudiantes de Alcalá se introdujeron en la corriente de lucha en la universidad española por la "ruptura democrática". Las intolerables condiciones en que se tenía que desarrollar la enseñanza $y$, contradictoriamente, con normas académicas más estrictas que en la Complutense, eran un buen ejemplo para los líderes estudiantiles de la necesidad de luchar por aquélla. A las autoridades del "Campus" se les hacía responsables de la situación "porque pertenecían a sectores reaccionarios del régimen desaparecido y a grupos de presión que pretendían hacer en Alcalá un experimento de universidad particularmente conservadora".

El movimiento estudiantil en Alcalá encontró, pues, un motivo de lucha en línea con los grandes temas que ocupaban a sus compañeros: había que evitar la conclusión de un experimento de aquellas características, exigiendo la dimisión de las autoridades y elecciones democráticas. Pero esto significaba una aceptación, de hecho, de que el "Campus" de Alcalá existía y de que debía ser como cualquier otra universidad española.

Tal como había sido la estrategia durante el franquismo, los movimientos reivindicativos de carácter político en la universidad partían de la lucha por la 
solución de aspectos concretos de la comunidad estudiantil en cada momento. Y por ello, en Alcalá, aquellas reivindicaciones generales se acompañaban, como conclusión lógica, de dos inmediatas que preocupaban individualmente a la mayor parte de los alumnos:

1. Mejora de las condiciones en las que se desarrollaba la enseñanza en el "Campus" (hubo, por ejemplo, más de una huelga exigiendo libros en la biblioteca), para lo que se hacía todo lo posible por que la opinión pública se enterara de la intolerable situación en la que tenían que estar en el "Campus" de Alcalá.

2. Vigencia de iguales normas que en "la Complutense", al considerarse estudiantes de la misma, y derecho a traslado a las facultades de la misma si así se deseaba.

De este modo, pudo dar la impresión de que los estudiantes y los profesores que les secundaban luchaban por el cierre de las instalaciones de Alcalá. De hecho, el Ministerio utilizó durante el Gobierno de Arias (siendo ministro Robles Piquer) el descontento estudiantil como argumento para expresar la necesidad de que los centros de Alcalá fueran cerrados y reenviados sus alumnos a Madrid.

Sin embargo, la propuesta de cerrar no apareció nunca en el proceso de lucha de los estudiantes y de los profesores más opuestos al grupo de dirigentes. En última instancia, fue este movimiento el que hizo que tomara carta de naturaleza en el mundo universitario el "Campus de Alcalá": en un comunicado de la asamblea de P.N.N. de las Universidades de Madrid, en febrero de 1976, apareció, por primera vez, una identificación de Alcalá separada de las otras Universidades de Madrid, aunque no se le encontrara otro posible nombre a las enseñanzas de Alcalá que "El Centro de Alcalá de Henares". 
En 1976, pues, la recién estrenada enseñanza universitaria de Alcalá tenía, como principal problema, el de su reconocimiento oficial por el Ministerio de Educación. Si las facultades eran creadas, su permanencia podría estar asegurada. La Universidad Complutense de Madrid, con un nuevo rector desde ese año (Vian Ortuño), no podía dejar crecer su problema de estudiantes indefinidos en Alcalá si permitía que, sucesivamente, las "facultades" abiertas de hecho fueran estableciendo los cursos siguientes o recibiendo nuevos estudiantes en los primeros cursos. Por otra parte, aunque así lo quisiera, no disponía de los créditos presupuestarios necesarios para el desarrollo del plan del "Campus" que ello hubiera requerido.

Los alcalaínos fueron nuevamente los que hubieron de movilizarse para salvar lo que tanto esfuerzo había costado conseguir; pero, una vez más, hay que resaltar que las acciones llevadas a cabo tuvieron detrás el especial entusiasmo y esfuerzo de personas concretas. Desde el Ayuntamiento, las concejalas Asela Sanz y Rosario Fernández Dovermans encabezaron la realización de multitud de gestiones ante las instancias gubernamentales, para obligarles a que se definieran sobre la extensión del "Decreto de creación" de las facultades en el "Campus" de Alcalá. Desde la sociedad pura y simple, Emilio Sanz, José García Saldaña, Sandalio San Román, Arsenio Lope, la Cámara de Comercio y las asociaciones culturales se constituyeron en Junta Gestora de una Sociedad de Amigos de la Universidad que realizó asimismo gestiones de tal tipo y con ellas demostró que el tema universitario en Alcalá contaba con el apoyo de todo tipo de fuerzas sociales y políticas.

Fue la disposición a luchar de esos grupos de alcalaínos, la que los responsables del "Campus" intentaron aprovechar para salvar la continuidad de las enseñanzas en él durante el curso 1976-1977, a pasar de la indiferencia del Ministerio de Educación y de los departamentos de la Universidad Complutense. 
La dirección del "Campus" planteó en una reunión de "las fuerzas vivas de Alcalá", en mayo de 1976, que el curso siguiente no se podía realizar en Alcalá y los estudiantes serían reenviados a Madrid si no se conseguían urgentemente 250 millones necesarios para acondicionar las instalaciones del "Campus". Esa inversión correspondía al compromiso inicial del Ministerio del que ya se había desentendido. Se les solicitaba que buscaran la forma de aportar tal suma.

Naturalmente que la realización de tan extraordinario esfuerzo era imposible para Alcalá, y mucho menos sin el concurso de la gran industria de la zona que, hay que decirlo, estuvo siempre al margen del proceso universitario. Pero sirvió de llamada de atención para que todas las instancias se movilizaran en torno al objetivo fundamental, cual era la consecución del decreto de creación.

La batalla por la creación de las facultades, no obstante, estuvo perdida en principio, de tal modo que al comenzar el curso 1976-1977 los estudiantes fueron readmitidos en las facultades correspondientes de Madrid y al profesorado no se le comunicó la continuidad de sus contratos. Sólo una negativa rotunda de las facultades de Medicina y Ciencias Económicas y Empresariales de Madrid de admitir de forma generalizada a los estudiantes de Alcalá hizo que, casualmente, tuviera que continuar la enseñanza de aquéllas, y sólo de aquellas enseñanzas en Alcalá durante el curso 1976-1977.

La Universidad, en principio, parecía desaparecida nuevamente de Alcalá antes del verano de 1976. El Ministerio incluso se negaba en mayo a recibir a una delegación del Ayuntamiento alcalaíno que quería saber cuáles eran las intenciones de aquél en cuanto a la creación oficial de los centros. A la corporación municipal no le quedó más remedio, para presionar, que intimidar con una dimisión en pleno de todos los concejales si la audiencia no se producía. 
Seguramente el tema alcalaíno se habría archivado, o como mínimo habría sido un nuevo proyecto temporalmente abortado, si no se hubiera dado aquella anómala circunstancia de que las facultades de la "Complutense" se negaran a considerar como propios los alumnos de Medicina y Económicas que habían cursado sus primeros estudios en Alcalá. La situación, que tan comprometida era para las autoridades del "Campus", permitió no obstante ganar el tiempo suficiente para que, sin cerrarse de hecho las instalaciones de Alcalá, las gestiones ante el Ministerio acabaran dando resultado.

El cambio de actitud del Ministerio de Educación con respecto al tema alcalaíno se produjo tras la constitución del primer Gobierno de Suárez (verano de 1976). Tal vez por la política de reconocimiento de la realidad de los conflictos y tensiones existentes en todas las esferas de la vida del país, con la tendencia a solucionarlos cediendo en lo que no se consideraba importante, o tal vez por propio convencimiento de las necesidades universitarias de Madrid, que le habían sido expuestas por el nuevo rector Vian Ortuño, el Ministerio cambió su actitud con respecto al tema universitario alcalaínoNota12 de VA. Por lo pronto reinició el dialogo con las instituciones alcalaínas al respecto, dando la esperanza de que no solamente serían creadas las Facultades, sino que se estudiaba la posibilidad de hacer del "Campus" una universidad independiente de Madrid.

Así, contradictoriamente, cuando en octubre de 1976 estaban cerradas las primitivas Facultades de Ciencias y Farmacia de Alcalá, por haberse devuelto sus estudiantes a Madrid, y se mantenían en precario Económicas y Medicina, se tenía, de nuevo, la esperanza de creación de la Universidad de Alcalá. Y por ello, el Ayuntamiento quiso agradecer al Rectorado de la "Complutense", en las personas del rector -Viany el vicerrector -Calvo- sus gestiones al respecto, otorgándoles una medalla denominada "del retorno de la Universidad". La esperanza había renacido de nuevo 
y, con ella, Felipe Calvo pudo expresar en el acto de concesión (9 de octubre de 1976):

La empresa que se avecina es dura, como las que gustaba afrontar el Cardenal [...] esta vez no suenan a cerrar, sino a abrir de par en par el Colegio y la ciudad a la nueva Universidad.

En diciembre de ese año, los alcalaínos pudieron escuchar por televisión, en boca del subsecretario de Educación (Martin Retortillo), que se iba a la creación de la Universidad de Alcalá. Pero escarmentados, no obstante, de tantas promesas incumplidas, todo el curso 1976-1977 estuvo pleno de gestiones, tanto del Ayuntamiento como de la Asociación de Amigos de la Universidad, para garantizar que lo verbal se tradujera en el correspondiente decreto.

Estas instancias estaban inquietas por la situación de protestas, paros y huelgas entre los estudiantes del "Campus", que ponían de manifiesto las deficiencias de las instalaciones del mismo y exigían:

a) Información sobre la posible independencia del "Campus" con respecto a la "Complutense", garantizándoles, en tal caso, los derechos adquiridos como alumnos de aquélla y, por tanto, el de escoger dónde quedar definitivamente.

b) Los mismos derechos que los alumnos de la "Complutense" en cuanto a normas de selectividad y permanencia en la universidad.

c) Participación en la redacción de los posibles estatutos.

d) Disposición de hospitales idóneos para la realización de prácticas de los estudiantes de Medicina. 
Las clases hubieron de suspenderse en varias ocasiones y los estudiantes, en la línea con lo que era habitual en el movimiento estudiantil de entonces, organizaron clases paralelas. También llegaron a organizar una manifestación con pancartas en Madrid, ante el Ministerio de Educación, exponiendo aquellas reivindicaciones y su oposición a que "el Opuis Dei estuviera interesado en crear aquí su universidad". Pedían, por ello, que la futura universidad, si se creaba, "estuviera financiada exclusivamente con presupuestos del Estado"Nota28.

La situación se podía calificar de frenética. Todo tipo de gestiones era válido. La Asociación de Amigos de la Universidad y la Sociedad de Condueños buscaron, y obtuvieron, el apoyo de la Diputación Provincial y los miembros en Alcalá de la recién creada $U C D$, procuraron hacer uso de sus relaciones ante personalidades de las instancias gubernamentales. Destacó especialmente la citada Rosario Fernández Dovermans, que pudo escuchar al fin, el 9 de junio de 1977, del gobernador civil de Madrid, Rosón, por llamada telefónica, el siguiente mensaje:

Mañana va por fin lo de la Universidad al Consejo de Ministros. Al fin te has salido con la tuya.

Esta vez si fue definitivo, de tal modo que por Real Decreto de 10 de junio de 1977 se creó una llamada "nueva universidad de Madrid con sede en Alcalá de Henares", es decir, una universidad realmente sin nombre definido, pero oficial al fin, y pudo celebrar Alcalá de Henares con todos sus pronunciamientos un acto en el Colegio de San Ildefonso, en el que el rector de la "Complutense" declaró:

En octubre del año pasado, con motivo de la apertura del curso actual, el Rector se comprometió públicamente a ello. Hoy siente el Rector la satisfacción -y la nostalgia- 
de su fidelidad. Aquí tenéis ya la Universidad, vuestra Universidad de hoy, para mañana y para el futuro.

Pero, ¿qué universidad era ésta, recién creada, dicho sea de paso, con una disposición de rango inferior (un Real Decreto), cuando lo legalmente establecido era haberlo sido con una Ley? El propio Decreto lo declaraba:

Nacida para resolver una situación de hecho:

La extraordinaria expansión [...] obligó en su día a la ubicación de alguno de sus centros [de la Complutense] en lugares alejados del núcleo central. Entre esos centros, los situados en Alcalá de Henares tienen ya, por la amplitud de las enseñanzas que se imparten [...] entidad suficiente para constituir una Universidad independiente...

Satisfacer las reivindicaciones de la zona:

Al mismo tiempo que enlaza con la rica tradición universitaria que dicha ciudad posee, supone establecer la enseñanza superior en un área de población en constante crecimiento.

Nacería progresivamente:

La nueva Universidad que se crea debe entrar en funcionamiento de modo gradual y progresivo: de ahí la vinculación que, sin perjuicio de su autonomía, se establece con la Universidad Complutense hasta que las dotaciones personales y materiales permitan su total independenciaNota13 de VA.

Pero con un gran conjunto de Facultades: 
Medicina, Ciencias Económicas y Empresariales, Farmacia, Derecho, Filosofía y Letras, en las que tenían que incorporarse las enseñanzas entonces cursadas en los Colegios Universitarios integrados en la Complutense.

Y el gobierno de la nueva universidad sería tutelado por la Complutense. mediante una Comisión gestora dependiente de la misma, hasta tanto que el Ministerio lo decidiera de acuerdo con aquélla.

Esta manera de ser creada la que sería "Universidad de Alcalá de Henares" (aunque nació sin tal nombre), llevaría en su germen una fuente de problemas que han lastrado el funcionamiento de la misma casi hasta nuestros días. En la práctica, la "apertura gradual y progresiva" significó abrir centros sin realizarse la inversión previa necesaria para dotarlos de los medios de infraestructura y equipamiento y sin hacer una planificación general de las dotaciones en medios humanosNota14 de VA. Desarrollo "progresivo" no significaba esperar a abrir la matriculación de los alumnos una vez que existieran los medios, sino abrir "progresivamente" los cursos subsiguientes de las enseñanzas existentes y de los primeros y sucesivos, cada año, de las demás y no comprometerse, por parte del Ministerio de Educación, a hacer la planificación e inversión necesarias de un proyecto acabado.

CONTINUARÁ...

En el próximo número: 1977-1978: los difíciles comienzos

\section{NOTAS}

NOTA 24: Ver Arriba, 2-8-75. 
NOTA 25: El "Plan Parcial del Campus de Alcalá" se acordó realizar por la Dirección General de Programación e Inversiones a propuesta de la Universidad Complutense, y se encargó a los arquitectos Blanch y Marquina, que habían redactado ya en 1974 el "Plan Parcial de la Ciudad Universitaria de la Moncloa".

NOTA 26: Estos tres informes se realizaron respectivamente en julio de 1975, en febrero de 1976 y en marzo de 1976, siendo el más completo el segundo de ellos.

NOTA 27: En los cursos 1975-76 y 1976-77 alcanzaron su punto más álgido las reivindicaciones universitarias en España, siendo más evidente que nunca antes la unión que había entre el movimiento estrictamente estudiantil, el de los P.N.N. (Profesores No Numerarios, los más jóvenes, con contratos profesionales muy precarios y pertenecientes en su mayoría a la generación "revolucionaria" del 68) y la consolidación de los partidos políticos de izquierdas, cuyos dirigentes eran en su mayoría universitarios jóvenes.

El Movimiento, en estos años, se concentró entorno a las siguientes reivindicaciones:

1. Amnistía universitaria. Es decir, readmisión de todos los profesores y estudiantes excluidos en los años anteriores por razones abierta o indirectamente políticas.

2. Democratización de la universidad. Es decir, ruptura democrática, con la iniciación de un proceso constituyente en el que participaran paritariamente profesores, alumnos y personal de administración y servicios, que estableciera formas de gobierno autónomas y participativas en la universidad.

3. Modificación de las normas de selectividad para el acceso y permanencia en la universidad. 
4. Eliminación del sistema de oposiciones para la selección del profesorado y su sustitución por un modelo basado en la contratación laboral.

5. Modificación de los planes de estudio de todas las carreras, con participación democrática de todos los sectores universitarios.

Desde la oposición democrática se consideraba que el primer Gobierno de la Monarquía y el primero de Suárez habían reducido la transición en la universidad a la permisividad de la libertad de expresión y reunión. Pero, sobre todo, con el segundo, se reconocía que había un intento de remodelar el viejo aparato educativo y, por tanto, que era el momento de forzar una aceleración en línea con la idea de la "ruptura democrática", para que la ya anunciada "autonomía universitaria" fuera algo verdaderamente progresista, democratizador y reformista. Para ello los partidos de izquierda y los sindicatos de enseñanza, con unos u otros matices, estaban de acuerdo en una serie de acciones que, de una forma $u$ otra, se pretendieron poner en práctica: unificación de los movimientos de estudiantes, de P.N.N. y de PAS (Personal de Administración y Servicios); puesta en funcionamiento de comisiones tripartitas redactadoras de estatutos en cada universidad; puesta en funcionamiento, de hecho, de consejos de departamento; entrada, antes de la redacción de los estatutos, de todos los sectores, en proporciones equivalentes, en los órganos de gobierno universitarios; boicot por parte del profesorado a las oposiciones; elaboración de un estatuto del profesorado; oposición al sistema de consultas iniciado por el Ministerio en torno a la problemática de selección y contratación del profesorado y a la creación de "comisiones oficiales" para la elaboración de estatutos.

NOTA 28: Diario 16, 5 de marzo de 1977. 
NOTA 5 DE VIVAT ACADEMIA: Volvemos a destacar la insistente idea de la ciudadanía alcalaína en recuperar no sólo la Universidad como Institución sino el carácter universitario de la ciudad, instalando centros en el casco antiguo. Sin embargo, dicha idea conllevaba la involucración directa de los responsables de los edificios en la planificación de Alcalá como ciudad universitaria, lo que parecía bastante razonable; prueba de ello es la propuesta de creación de instituciones comunes.

NOTA 6 DE VIVAT ACADEMIA: En muchos de sus planteamientos, el profesor Calvo fue un adelantado a su tiempo; y sus ideas, pese a su fama de conservador, fueron puestas en práctica, para toda la universidad española, al menos sobre el papel, por la reforma socialista plasmada en la LRU. Sin embargo, cometió un error gravísimo, del que todavía nos resentimos, fue el pretender que la Universidad de Alcalá formara exclusivamente futuros docentes, sobre todo en el ámbito de las enseñanzas medias. Para ello debería haber existido libre elección de universidad por parte de los estudiantes e información precisa de los estudios implantados, ya que aquéllos venían obligados por las circunstancias y se encontraban que una licenciatura de las de Alcalá era muy diferente de las existentes en cualquier universidad del país. Los nuevos planes de estudio alcalaínos todavía han heredado, en algunos casos por razones de simple inercia, muchos defectos de los impuestos por Felipe Calvo. En otros, por razones de rechazo a lo anterior (el llamado efecto péndulo), han caído en los errores propios del "lado opuesto".

NOTA 7 DE VIVAT ACADEMIA: Conviene resaltar aquí, para los que no han vivido estos hechos, la planificación que para el "Campus de Alcalá" preveía Felipe Calvo. En demasiadas ocasiones hemos oído al rector Gala atribuirse las iniciativas de construcción de residencias y jardín botánico, haciéndolas un proyecto "personal e innovador", cuando son herencia de las ideas pioneras de los que hicieron posible la resurrección universitaria alcalaína, independientemente de que sean acertadas o no. 
NOTA 8 DE VIVAT ACADEMIA: Estas instalaciones corresponden a los dos edificios laterales de la actual Facultad de Ciencias. El edificio central era un proyecto de edificación, que sólo constaba de los cimientos y columnas de planta baja en hormigón. Además, existían la "Torre de Control" de la Base Aérea, actual centro de Cálculo, y un pequeño edificio, donde se ubicaría la Facultad de Medicina, hoy Departamento de Biología Celular y Genética.

NOTA 9 DE VIVAT ACADEMIA: Los pioneros que viajaban en tren en aquella época recuerdan, con horror, la inexistencia del andén del apeadero, debiéndose bajar en pleno campo. Eso en el mejor de los casos, porque muchas veces el maquinista olvidaba que debía parar en aquel descampado y alumnos, profesores y PAS se veían en Meco, con horas por delante hasta el próximo tren procedente de Guadalajara, ya que no todos tenían parada en Alcalá Universidad. Esta situación continuó produciéndose con relativa frecuencia incluso varios años después de la construcción del apeadero. Sería interesante revisar los libros de reclamaciones de esos años, donde muchos han plasmado su firma indignados, sabedores de que nadie le pondría fin a tan desagradables hechos.

NOTA 10 DE VIVAT ACADEMIA: Pero nosotros entendemos que eso, precisamente, era lo que menos garantía representaba, ya que los alumnos, lejos de estar contentos con estudiar en una de las más grandes antaño universidades de España, sabían, dado como se cuecen las cosas en nuestro país, que la calidad no sería buena y el viaje diario resultaba verdaderamente penoso. Por estas razones estaban deseando que se cerraran los estudios en Alcalá y les devolvieran a Madrid. Y son los estudiantes los que tienen fuerza cuando se trata de hacer presión.

NOTA 11 DE VIVAT ACADEMIA: En la actualidad eso es cierto sólo sobre el papel de la ley escrita. En la práctica siguen existiendo en la universidad española 
pequeños grupos de presión, alentados, cuando no creados, por los propios rectores, con el fin de mantenerse en el poder, muchas veces para sacar adelante proyectos peregrinos de connotaciones puramente personales. Los claustros han sido prácticamente vaciados de todo contenido y las juntas de gobierno funcionan, mucho más que antes, al son que le tocan los equipos rectorales. La perversión del sistema democrático, ayudada por la desidia y el mercantilismo de muchos de los profesores y demás trabajadores de la universidad, hace posible cualquier cosa, por lejana que esté del bien de la comunidad.

NOTA 12 DE VIVAT ACADEMIA: Nosotros tenemos otra teoría al respecto. Una vez que se planteó la llegada al poder del Estado, la UCD sentía la necesidad de acercar hacia la capital a sus militantes y simpatizantes universitarios desperdigados por el país. Las otras universidades madrileñas estaban lo suficientemente saturadas de profesorado, sobre todo de catedráticos y agregados, como para no poder permitir una operación de "desembarco". La posibilidad de abrir una nueva universidad en el centro permitiría reagrupar a los líderes del partido que se formaba. De hecho, en la Facultad de Derecho de la Universidad de Alcalá (y en la de Económicas y Empresariales, aunque en menor medida), al poco de la publicación del Real Decreto de su creación, se dotaron, casi al completo, las plazas de profesores numerarios, que fueron siendo ocupadas por destacados miembros de la UCD, que paulatinamente se han ido trasladando a las Universidades de Madrid. Algo parecido, al margen de necesidades académicas, han hecho posteriormente el PSOE y el PP con la creación, respectivamente, de las Universidades Carlos III y Rey Juan Carlos.

NOTA 13 DE VIVAT ACADEMIA: De hecho todo seguía dependiendo de la "Complutense", como ejemplo, diremos que los profesores que eran contratados, (los numerarios estaban todos en comisión de servicios), debieron formalizar sus 
contratos en el Rectorado de la Universidad Complutense, hasta mucho después de la publicación del Real Decreto.

NOTA 14 DE VIVAT ACADEMIA: Ese problema no es debido a la época en que se realizó la reapertura, sino, en la mayoría de los casos, a las necesidades de ciertos grupos de presión que funcionan en connivencia con las autoridades académicas. Hemos asistido en los últimos años a una creación frenética de nuevos centros en la Universidad de Alcalá, que adolecen de los mismos problemas, cuando no mayores. 